\title{
A Radial Basis Function Based Approach for Polygon- free Reconstruction and Representation of 3D Objects from Range Data
}

\author{
（レンジデータからの 3 次元物体の再構成と表現のための Radial Basis Functionに基づいた手法）
}

\author{
Hamid Laga ${ }^{\dagger}$, \\ Romanos Piperakis $^{\dagger \dagger}$, Hiroki Takahashi ${ }^{\dagger}$, \\ Masayuki Nakajima (member) $)^{\dagger}$
}

\begin{abstract}
The acquisition of the 3D shape of real objects is a rapidly expanding field with various applications in Computer Aided Design, archeology, design, entertainment and so on. In this paper we propose a new approach for 3D object reconstruction from range data. Our approach is based on the modelization of the object surfaces with a set of RBFs (Radial Basis Functions). Our originality is the fact that the reconstruction is independent from the object geometry ; it can be applied for smooth objects as well as for objects with sharp boundaries. To enhance the efficiency of the RBF modelization, we propose also an efficient isosurfacing algorithm. Unlike conventional reconstruction techniques, no prior information about the acquisition process is required. We demonstrate the ability of our approach to reconstruct a wide range of 3D objects through a set of experiments.
\end{abstract}

Key words: Radial Basis Function, surface representation, implicit surfaces, 3D Reconstruction, Isosurfacing.

\section{Introduction}

Advanced computer graphics applications require detailed 3D models of their environment. The desire to reduce the dependency on the human input in making realistic 3D models of real complex scenes has resulted in an increased role of the world measurement in the computer graphics society.

Recent growth in implicit surface representations with their foundations established early in the development of 3D curves and surfaces in computer graphics ${ }^{3)}$, has created new interest in polygon free reconstruction and representation of $3 \mathrm{D}$ objects and scenes from a set of scattered data. This was also motivated by the recent advances in CPU speeds, available memory and computing costs. However, managing complex models remains difficult. Early works in implicit models use a combination of boolean operations and blending functions to describe CAD objects ${ }^{12)}$. These techniques are

Reported in NICOGRAPH2002, (2002.10.25)

Received October 31, 2002; Revised July 22, 2003; Accepted September 4,2003

† Graduate School of Information Science and Engineering, Tokyo Institute of Technology

(2-12-1, Ookayama Meguro-ku 152-8552, Tokyo, Japan )

$\dagger †$ Precision \& Intelligence Laboratory, Tokyo Institute of Technology ()

$1526(128)$ suitable for object description rather than reconstruction. Piecewise parametric splines have also been used to define surfaces implicitly. An introduction to these techniques can be found in the paper ${ }^{12)}$.

J.C.Carr et. al. ${ }^{2)}$ have developed techniques for modelling and reconstructing 3D objects from surface point data using a set of RBFs (Radial Basis Functions). This work provides some insight into how one implicit function can be fitted to complex and smooth objects. It allows a direct specification of a complex surface from dense, irregular data samples. However, their approach is adapted only for smooth objects without sharp boundaries. More ever, only a random subset of the initial scarred points is used to fit the implicit function. Although this optimization reduces dramatically the fitting time and the required memory, a better result could be obtained by using prior knowledge about the scene to model or statistical analysis on the initial data set.

On the other hand, to reconstruct sharp features such as edges and corners, super-quadratic surfaces are widely used ${ }^{13)}$. Although it allows to approximate sharp features, the resulting surface remains mathematically smooth at the sharp boundaries.

This paper introduces a new approach for 3D Object modelling and reconstruction from range data. First, 
the object surface is fitted with a set of RBFs. The merit of our technique is the fact we don't make any restriction on the object geometry. This technique is coupled to an efficient isosurfacing algorithm which will allow us to extract with a high precision the mesh representation of the scene from the RBF based implicit representation. As we will show in the next sections, our approach is fully automatic, and no prior knowledge about the acquisition system is required. However, in this paper, the range data acquisition problem is not considered. This problem is well studied in many papers ${ }^{18) 9)}$. In our work, we consider that the 3D range data are already available. The distinctions between our work and the well-known techniques are:

(1) The entire object is modelled with a single implicit function.

( 2 ) No restriction is made on the object geometry: the approach is adapted for smooth objects as well as objects with sharp boundaries.

( 3 ) For a fast fitting of the RBF to the data set, unlike the previous method $i^{2)}$, the center reduction technique is not random but uses a statistical analysis on the initial data. This gives a powerful optimization tool.

(4) In the iso-surface extraction step, the range data are used in order to localize the surface. This avoid extra evaluations of the implicit function and results in less computational complexity.

In section 2, we will introduce our technique for surface fitting using RBFs and explain how it can be optimized in order to be able to fit any given set of points. Section 3 will discuss and propose a solution to the problem of surface extraction from an implicit representation. Some experimental results and evaluation are presented in section 4 , and we will terminate by a conclusion future work.

\section{Surface representation}

\subsection{Implicit representation}

Given a set of $n$ scanned $3 \mathrm{D}$ points $S=\left\{s_{i}=\right.$ $\left.\left(x_{i}, y_{i}, z_{i}\right), i=1 \ldots n\right\}$, the problem is to find a surface $M$ which fits the points in $S$. A common and efficient analytical representation of the surface $M$ is to use an implicit function $f$. Thus, every point $s=(x, y, z)$ belonging to $M$ has to satisfy the following equation;

$$
f(x, y, z)=0 .
$$

An efficient way is to choose $f$ as the signed distance of a point $(x, y, z)$ to the closest point on M. In our case, we wish to find a function $f$ which is zero for every point in the set $S$;

$$
f\left(x_{i}, y_{i}, z_{i}\right)=0, \quad i=1, \ldots, n .
$$

In order to avoid the solution that $f$ is zero everywhere, we add off-surface points to the original data;

$$
f\left(x_{i}, y_{i}, z_{i}\right)=d_{i} \neq 0, i=n+1, \ldots, N .
$$

An off-surface point is generated for each point $s \in S$ along its normal to the surface and in both directions. The normal vectors are calculated using the Principal Component Analysis on a restricted neighborhood of each point. Determining a neighborhood of a pixel is an important problem especially for a huge data set. In our approach, when the system is used to reconstruct an unorganized set, a preprocessing step is added. In this step, a Kd-tree structure is constructed and thus the neighborhood of any point can be found in $O(n \times \log (n))$ instead of $O\left(n^{2}\right)$. We will show later that this structure is also very useful to speed up the evaluation of the RBF during the isosurface extraction process. From this point forward, we will use the symbol $S$ to denote the union of the sensed points and the computer generated off-surface points.

\subsection{Function approximation}

Many classes of functions have been proposed to address the problem of fitting the points in $S^{2)^{6) 12)}}$. A linear sum of RBFs has the advantage of representing the whole surface with a unique function. Using an appropriate radial basis, we can express the interpolation function as;

$$
f(s)=p(s)+\sum_{i=1}^{N} \lambda_{i} \phi\left(s-s_{i}\right) .
$$

where $\Delta=\left(\lambda_{1}, \ldots, \lambda_{N}\right)^{t}$ a vector of $N$ real parameters to determine. The model involves a first degree polynomial $p$ which stabilizes the process:

$$
p(s=(x, y, z))=c_{1}+c_{2} x+c_{3} y+c_{4} z .
$$

where $C=\left(c_{j}, j=1 \ldots 4\right)$ are real coefficients to estimate and $\phi$ is the basic function. On an initial inspection, the compactly supported basic functions appear to lead to more desirable properties ${ }^{3)}$. However, they are not adapted for interpolating irregular, nonuniformly sampled data. J.C.Carr et. al. ${ }^{2)}$ propose to use a biharmonic spline $\left(\phi(r)=\sqrt{r^{2}+c}\right)$, because of their energy minimization properties and their ability to interpolate and extrapolate where the data are missing. However, these functions are adapted only for objects with smooth surfaces. In our work, we propose an 

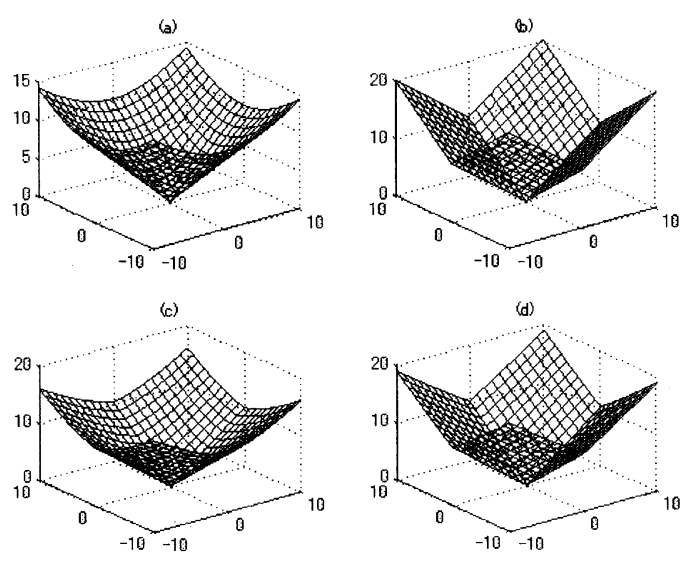

Fig. 1 The basic function used in the interpolation. (a) A biharmonic spline. (b) A rectangular function. Its non-derivative property allows to model boundaries. (c) A linear combination of the two basic functions, $\tau=0.8$ the boundaries are smooth. (d) $\tau=0.2$, the boundaries are sharp.

adapted function for smooth surfaces as well as surfaces with sharp boundaries. First, we introduce a new real parameter $\tau, \tau \in[0, \ldots, 1]$ which controls the surface smoothness. Thus, a value 1 of $\tau$ is used for smooth surfaces and the value 0 is used for objects with sharp boundaries. Now, the radial basic function becomes;

$$
\phi(r)=\tau \phi_{1}(r)+(1-\tau) \phi_{2}(r) .
$$

where $r=s-s_{i}, s=(x, y, z)$ is a $3 \mathrm{D}$ point and $s_{i} \in S, s_{i}=\left(x_{i}, y_{i}, z_{i}\right)$ is the basic function center. The positive real function $\phi_{1}$ is defined in order to control the surface smoothness, and the biharmonic spline is the most adapted for this purpose;

$$
\phi_{1}(r)=\sqrt{\left(x-x_{i}\right)^{2}+\left(y-y_{i}\right)^{2}+\left(z-z_{i}\right)^{2}} .
$$

Now the second term of the equation (6) controls the surface sharpness. In our work, we propose to use the rectangular function defined as;

$$
\phi_{2}(r)=\left|x-x_{i}\right|+\left|y-y_{i}\right|+\left|z-z_{i}\right| .
$$

The behavior of these two functions and their linear combination, i.e $\phi(r)$, is illustrated in Fig. 1.

Fig. 2 is a synthetic example of the reconstruction of a noisy cube from 1437 sample points. To better illustrate how the Parametric RBFs model approximates general boundaries, the cube is not aligned to any of the coordinate system axis. For the experiment, the value 0.05 of $\tau$ is used. In this method, $\tau$ is set manually. The automatic estimation is a very complex problem and will be a subject of future work. Fig. 2(a) shows the reconstruction of the same cube using the thin-plate described in ${ }^{2)}$, while the reconstruction in Fig. 2(b) and (c)

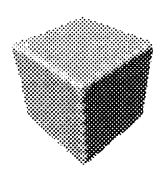

(a)

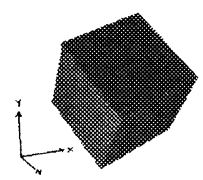

(b)

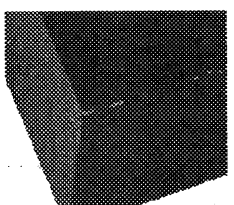

(c)
Fig. 2 Thin-plate (a) and Parametric RBF ((b) and (c)) Reconstruction of a noisy synthetic cube from 1437 sample points with $\tau=0.05$. (c) shows a zoom on one of the cube corners.

is achieved using our new model. The thin-plate model produces rounded edges and corners. In contrast, the reconstruction using Parametric Radial Basis Functions exhibits sharper edges and corners, and more faithfully reproduces the polygonal cube whatever is the boundary direction. This also demonstrates the applicability of the model to general boundaries.

Now, we need to estimate the real parameters $\Lambda=$ $\left(\lambda_{1}, \ldots, \lambda_{N}\right)^{t}$ and $C=\left(c_{1}, c_{2}, c_{3}, c_{4}\right)^{t}$. From equations (2), (3) and (4), validated for each of the $N$ points, we get $N$ linear equations. By incorporating the orthogonality conditions ${ }^{4}$, it leads to a linear system to solve;

$$
\left(\begin{array}{ll}
A & P \\
P^{T} & 0_{4 \times 4}
\end{array}\right)\left(\begin{array}{l}
\Lambda \\
C
\end{array}\right)=B\left(\begin{array}{l}
\Lambda \\
C
\end{array}\right)=(D) .
$$

where $A$ is a $N \times N$ matrix such as $A_{i, j}=\phi\left(s_{i}-s_{j}\right)$, $P$ is a matrix with the $i-t h$ row equal to $\left(1, x_{i}, y_{i}, z_{i}\right)$, $0_{4 \times 4}$ is a $4 \times 4$ zero matrix and $D=\left(d_{1}, \ldots, d_{N}\right)^{t}$ the function values at the points in $S$. $B$ is a dense $(N+4) \times(N+4)$ symmetric matrix and has no apparent structure other than symmetry. Solving directly the system is time and memory consuming especially when the number of points to fit exceeds 10,000 . To overcome this problem we introduce two optimization stages, one during the fitting process, and the other during the isosurface extraction step.

\section{3 Fast estimation of the function parame- ters}

The objective in this step is to develop a fast evaluation method for solving the equation system (9). Here we present only the application for RBF and the results. The reader is referred to ${ }^{4) 5)}$ for the related theory.

The first idea is to define another basis for the subspace $\mathcal{F}$ of fitting functions:

$$
\mathcal{F}=\left\{f, f(s)=\sum_{i=1}^{N} \xi_{i} K\left(s, s_{i}\right)\right\} .
$$

where $K$ is the basic function, $s_{i}$ the points to fit and $\Xi=\left(\xi_{1}, \ldots, \xi_{N+4}\right)^{t}$ the RBF parameters to estimate. If $x, y$ are two variables in $\Re^{3}$, the basic function $K$ is 


$$
\begin{aligned}
& K(x, y)=\phi(x, y)+\sum_{i=1}^{l} \sum_{j=1}^{l} \delta_{i}(x) \delta_{j}(y) \phi\left(x_{i}-x_{j}\right)- \\
& \sum_{j=1}^{l} \delta_{j}(y) \phi\left(x-x_{j}\right)-\sum_{i=1}^{l} \delta_{i}(x) \phi\left(x_{i}-y\right) \\
& +\sum_{i=1}^{l} \sum_{j=1}^{l} p_{i}(x) p_{j}(y) .
\end{aligned}
$$

$\delta_{j}, j=1 \ldots l$, the delta function defined as $\delta_{j}\left(x_{i}\right)=1$ if $i=j$ and 0 otherwise, are Lagrange basis. The linear system to solve becomes:

$$
\left(\begin{array}{ll}
I & 0_{l \times(m-l)} \\
E^{T} & C
\end{array}\right)(\Xi)=E(\Xi)=(D)
$$

where $I$ is a $l \times l$ identity matrix, $\mathrm{C}$ is the ma$\operatorname{trix}\left(K\left(s_{i}, s_{j}\right)\right)_{i, j=l+1}^{m}, E^{T}$ is the $(m-l) \times l$ ma$\operatorname{trix}\left(\delta_{j}\left(s_{i}\right)\right)_{i=1 \ldots l, j=1 \ldots(m-l)}, m=N+4$ and $\Xi=$ $\left(\xi_{1}, \ldots, \xi_{N+4}\right)^{t}$ are the new parameters to estimate. First, we solve for the first $l$ coefficients, then by substitution and Singular Value Decomposition, we can solve for the remaining parts of the system.

This new linear system is easier to solve than (9), because the matrix $E$ is well-conditioned; it is sparse and contains identity submatrix. Compared to direct methods which require $O\left(N^{2}\right)$ of storage and $O\left(N^{3}\right)$ of flops which is impractical for large problems, solving the linear system (12) requires only $O(N)$ storage (since it is sparse) and $O(N \log N)$ for solving.

The second idea is to reduce the points used to estimate $f$ since the human perception is insensitive to small errors and scanning devices provide an over sampling of the object which results in redundant points. A typical example is a scanned plan where we get a large number of points while only few suffice to define the whole plan.

Taking advantages of the inherent statistical redundancy of the points in $S$, and by allowing a certain error to the fitting process, we choose to use a representative sample of the points instead of the entire set. If $N$ is the cardinality of $S$ and $n$ is the cardinality of the chosen set, then this leads to significant computational advantages in the case where $n \ll N$. The optimization process can be summarized as follow:

(1) Select a subset $A$ of $S$ as follows; for objects with sharp boundaries: if a point $s$ and the set of its neighbors are lying approximatively on the same plan then they are reduced to 3 points only. For objects without any geometric properties, we choose randomly the subset $A$.
(2) Fit the RBF to the subset A and evaluate the error at the points in $S-A$.

( 3 ) If the error is bigger than a threshold value $\varepsilon$ then we add new points to $A$ and we repeat the process from 2. Otherwise, we terminate the fitting process.

The point selection algorithm uses a Kd-tree structure created during the preprocessing step. This technique allows the user to compromise between fitting quality and processing time. Note that other criteria for point selection can be used and this approach doesn't lead to any inconsistency due to an inadequacy of the selection criteria with the scene geometry structure, because the error estimation and new point adding allow to compensate for any possible error generated at the early steps.

\section{Isosurface extraction}

A RBF fitted to a set of surface points represents a solid model of the object and its surface is the locus of points where the RBF is zero. In this section, we focus on the visualization of a $3 \mathrm{D}$ model represented by a set of RBF, known as Isosurface extraction.

In order to render the scene, a polygon representation is generated from the implicit function. This can be done using well known isosurfacing algorithms such as Marching Cubes or Marching Tetrahedra ${ }^{10) 11)}$. In these techniques, the isosurface is extracted by evaluating the implicit function on a grid covering the objects space. Since we are using radial basis functions, the complexity of the implicit function evaluation at a given point is $O(N)$, where $N$ is the number of the RBF centers, and for the entire 3D space, it becomes $O\left(r^{3} \times N\right)$, where $r$ is the resolution we use for the visualization.

In order to speed up the process and thus, avoid additional evaluations of the function in regions which doesn't contain any surface point, we propose a new isosurface extraction algorithm which is a volumetric based approach. Our idea is to use the range data themselves in order to localize the object surfaces, then evaluate, in the localized area, the RBF in order to get a more accurate localization. As we will see, this approach speeds up the RBF evaluation and the polygon extraction.

\section{1 Surface localization}

The object surface is localized in a hierarchical fashion. First we start by determining the scene's bounding box then consider it as the root of the octree. Then each octree node is classified as boundary, a voxel which intersects the object surface, or non-boundary, as illus- 


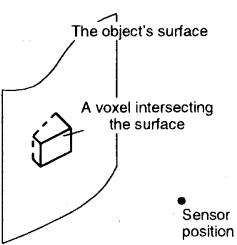

(a)

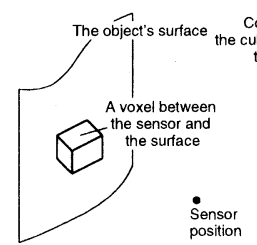

(b)

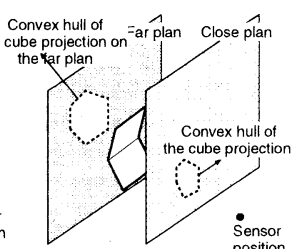

(c)
Fig. 3 Voxel Classification. (a) A boundary voxel. (b) A non boundary voxel. (c) Voxel classification criteria.

trated in Fig. 3. The boundary nodes are again subdivided and the process is repeated until we reach the finest level of subdivision. Then, the implicit function, given by the equation (4), is evaluated at the vertices of each leaf of the octree classified as boundary. The polygon meshes are generated using the traditional Marching Cube method. This approach has two main advantages over the previous ones ${ }^{10) 11)}$. In fact, during the subdivision and classification process, the RBF is not evaluated. Indeed, the geometric properties of the $3 \mathrm{D}$ object and information about the acquisition system, if available, are used as classification criteria. The second advantage is the fact that it allows the incorporation of some information about the acquisition system, when available, thus it makes the approach more general.

\subsection{Node Classification}

Node classification is the most critical step in the isosurface extraction process; each node in the octree is classified into boundary or non-boundary.

When the voxel is located between the view point and the surface, or far away it is classified as a nonboundary node. Otherwise the voxelit will classified as a boundary node. Fig.3(c) illustrates the classification criteria. Let, $V$ denotes the current voxel (or node), $c_{1}$ and $c_{2}$ are, respectively, the closest and the farthest vertex to the view point $v$. The plane $P_{1}$, we call the closest plane, containing the vertex $c_{1}$ and perpendicular to the view direction. Similarly, $P_{2}$ denotes the farthest plane. The classification process is as follows;

(1) Project perspectively the voxel vertices on the plane $P_{1}$ along the view direction, and calculate the $2 \mathrm{D}$ convex hull.

( 2) For all points projecting inside the convex hull, if some of them are located between the planes $P_{1}$ and $P_{2}$ then $V$ is classified as boundary.

( 3 ) Otherwise $V$ is classified as non-boundary. When the acquisition process either does not provide information about the view point, or we have no prior knowledge about the process at all, which is the case in our experiments, the algorithm selects one randomly. In the case where the sensor positions during the scanning are known, they are used in the reconstruction process. Note no restriction is made on the sensor position. The algorithm works even when the sensor is inside the object, because during the subdivision process, when we reach the finest level, the voxels containing the sensor position point are eliminated from the octree.

\section{Experimental results}

We have tested our method on two types of real data; smooth objects and objects with sharp boundaries as illustrated in Figs. 4-6. In our experiments, the smoothness parameter $\tau$ is set manually and remains constant for all the RBF centers. Thus, for bunny data, since it is a smooth surface, $\tau$ is set to 1.0. The objects in Figs. 5, 6 contain more sharp boundaries, and the values 0.5 and 0.1 , respectively, are found to be more suitable.

Table 1 summarizes the range data characteristics and the algorithm parameter setting : the tolerated fitting error $\varepsilon$ and the smoothness parameter $\tau$. It shows also the results of fitting a RBF to those data.

For the objects in Fig. 5 and Fig. 6, we used the point

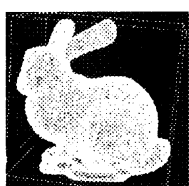

(a)

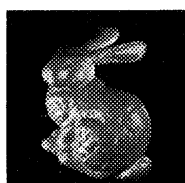

(b)

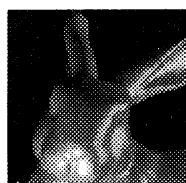

(c)
Fig. 4 Reconstruction of the Stanford Bunny, $N=$ $138,902, \tau=1.0, \varepsilon=1 \%$. (a) Input range data (b) The generated surface. (c) A view of a small part of the generated surface.

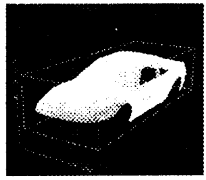

(a)

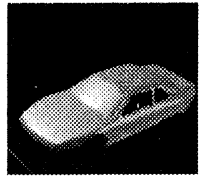

(b)

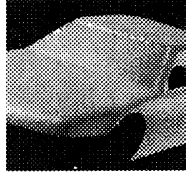

(c)
Fig. 5 Reconstruction of an object with boundaries: a car, $N=35,947, \tau=0.5, \varepsilon=1 \%$. (a) Input range data. (b) the generated surface model. (c) A detailed view of the generated surface.

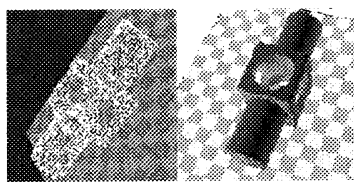

(a) (b)

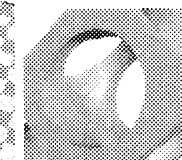

(c)

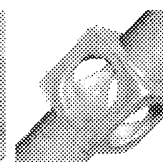

(d)
Fig. 6 Reconstruction of an object with sharp boundaries: $N=4,102, \tau=0.1, \varepsilon=1 \%$. (a) Input range data. (b) The generated surface. (c) A detailed view of the reconstructed surface. (d) Reconstruction using super-quadratic surfaces. 
Table 1 Fitting 3D object with PRBF, $\varepsilon=1 \%$, implemented on an Athlon 1.1Ghz, 128MB of RAM. The total interpolant points is the number of on-surface plus the off-surface points.

\begin{tabular}{c|c|c|c|c|c|c}
\hline \hline Data & $\begin{array}{c}\text { Scanned } \\
\text { points }\end{array}$ & $\tau$ & $\begin{array}{c}\text { Total } \\
\text { interp. } \\
\text { points }\end{array}$ & $\begin{array}{c}\text { RBF } \\
\text { Centers }\end{array}$ & time & error \\
\hline Fig.4 & 138,902 & 1.0 & 390,314 & $87,347(22.3 \%)$ & $1.095 \mathrm{~s}$ & $1.12 \%$ \\
\hline Fig.5 & 35,947 & 0.5 & 103,167 & $21,363(20.7 \%)$ & $207 \mathrm{~s}$ & $0.89 \%$ \\
\hline Fig.6 & 4,102 & 0.1 & 12,284 & $2,713(22.0 \%)$ & $23 \mathrm{~s}$ & $0.47 \%$ \\
\hline
\end{tabular}

Table 2 Effect of the fitting threshold $\varepsilon$ on the fitting process. The input data are from Fig.5.

\begin{tabular}{c|c|c|c}
\hline \hline$\varepsilon$ & RBF centers & fitting time & error \\
\hline $0.5 \%$ & $32,729(31.7 \%)$ & $478 \mathrm{~s}$ & $0.47 \%$ \\
\hline $1 \%$ & $21,363(20.7 \%)$ & $207 \mathrm{~s}$ & $0.89 \%$ \\
\hline $2 \%$ & $18,048(17.5 \%)$ & $143 \mathrm{~s}$ & $1.92 \%$ \\
\hline
\end{tabular}

reduction technique mentioned in section 2.3 because CAD objects contain many facets and can be approximated to planes. However we didn't use such optimization for the reconstruction of the Stanford Bunny in Fig. 4, the initial subset is selected randomly.

We can see in Table 1 that the RBF centers are reduced to almost $20 \%$ of the on-surface and off-surface points while the fitting error remains acceptable. Although our reduction ratio is less than the method proposed in ${ }^{2)}$ where the centers are reduced to $12 \%$, our algorithm is much more faster. This is due to the fact that our center selection method is deterministic, thus the suitable centers are selected in less than 3 iterations, while $i^{2)}$ many iterations are required since the selection is random.

Table 2 illustrates the effect of the fitting threshold $\varepsilon$ on the final result and the fitting time, for the range data in Fig.5. In fact, choosing a small value of $\varepsilon$ will reduce the fitting error while it increases the processing time. The setting of $\varepsilon$ is left to the user in order to balance between processing time and fitting quality.

Fig. 5(b) and (c) show the generated surface from the range data of Fig. 5(a). In Fig. 6 an object with very sharp boundaries is reconstructed using the Parametric Radial Basis Functions. The result is illustrated in

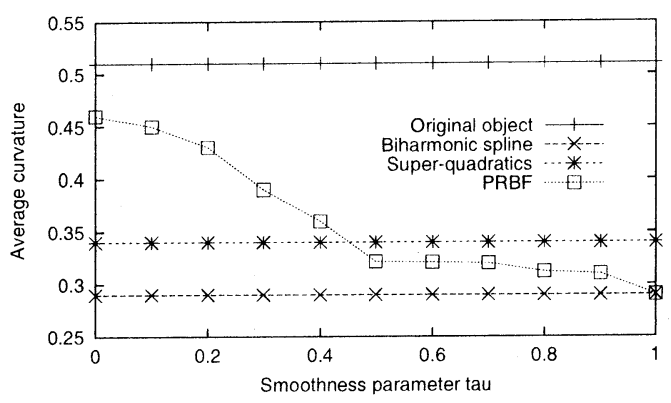

Fig. 7 Comparison of the average curvature
Fig. 6 (b) and (c). Unlike the reconstructed object using super-quadratic surfaces ${ }^{13)}$ in Fig.6(d), we can see that sharp features are preserved with our model. A zoom on the surface shows clearly, in Fig. 6(d), that with super-quadratic surfaces sharp features are approximated to a smooth surface while our model, as illustrated in Fig. 6(c), recovers the sharp feature.

To demonstrate our result, we compare the reconstruction quality at the sharp features for two conventional methods. In our experiment we use a curvature measure to evaluate efficiently the surface sharpness since sharp features can be distinguished by their high curvature, while low curvature is associated with overshoots and smooth surfaces. It is calculated based on the normal directions of triangles adjacent to each vertex and normalized by the total area of the triangles ${ }^{14}$. The plot in the Fig. 7 shows the average curvature at the sharp features at different values of $\tau$ for the biharmonic spline (by setting $\tau=1$ ), super-quadratic functions and our method. Because the two former methods are not dependent on $\tau$ the curvature is constant. We can see clearly that for large values of $\tau$, the Parametric RBF smoothes the surface. However by setting $\tau$ to values close to zero the curvature is high thus, the sharp feature is recovered with high quality. Compared to the original object, for $\tau=0.1$, the curvature error for our method is 0.06 while using super-quadratic surfaces the error is 0.17 .

Moreover, a super-quadratic function requires two parameters to model the surface sharpness, while using the PRBFs, only one parameter, $\tau$, is required. This simplifies significantly the problem of the automatic estimation of the model parameters. Other results are shown in Fig. 8.

Finally, direct solving of the PRBF fitting problem of the bunny and Budha data in Fig. 4 and Fig. 8 respec-

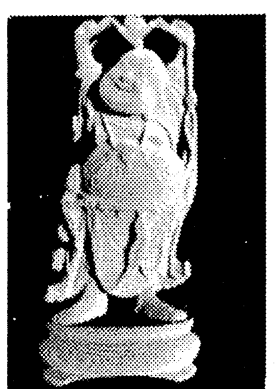

(a)

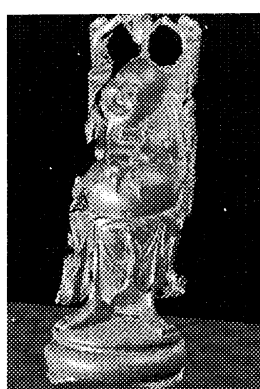

(b)
Fig. 8 Buddha surface reconstruction from range data (a) The input range data, $n=543,652, \tau=1.0$. (b) The reconstructed surface. 
Table 3 Processing time comparison between the previous approach ${ }^{2)}$ and the proposed approach. The processing time is given in hh:mm:ss

\begin{tabular}{c|c|cc|cc}
\hline \hline Data & $\begin{array}{c}\text { Scanned } \\
\text { points }\end{array}$ & $\begin{array}{c}\text { RBF } \\
\text { centers }\end{array}$ & $\begin{array}{c}\text { Total } \\
\text { time }\end{array}$ & $\begin{array}{c}\text { RBF } \\
\text { centers }\end{array}$ & $\begin{array}{c}\text { Total } \\
\text { time }\end{array}$ \\
\hline Fig.8 & 543,652 & 80,518 & $4: 03: 26$ & 123,743 & $01: 19: 24$ \\
\hline
\end{tabular}

tively, would require more than $30 G B$ of RAM and inestimable computational time $\left(O\left(N^{3}\right)\right)$. But using our method which makes a transformation to the Lagrange basis, the observed RAM peak is $87.3 M B$ for the bunny data. Table 3 shows a comparison of the computational time between our method and the one proposed in $^{2)}$ using the range data of the happy Budha statue. This table shows clearly that our method is much faster than previously proposed ones. This demonstrates the efficiency of the optimization techniques we introduced.

\section{Conclusion and future work}

In this paper we presented a new approach for 3D object reconstruction from range data. The coupling of RBF representation with an efficient isosurface extraction algorithm has improved the reconstruction results. We have shown through the examples the ability of the method to construct objects with sharp boundaries as well as smooth objects. This is achieved by introducing the smoothness parameter $\tau$ and a new radial basic function well adapted for the object constraints. Also the method is completely automatic and flexible since information about the acquisition process, if available, can be incorporated into the algorithm to yield better and faster results.

As future work, it is interesting, for a same object, to set the smoothness parameter $\tau$ to different values for different centers: a value close to zero for centers located near sharp boundaries, and a value close to 1 for centers located near the smooth parts of the surface. Although the accurate automatic estimation is a very complex problem, we are planning to control the smoothness parameter using the Principal Component Analysis for every center on its restricted neighborhood. The resulting eigenvectors are an efficient measure of the surface sharpness at a given center. This will allow more control over the surface topology.

Finally in this work we did not consider a mesh optimization step. Reducing the number of the generated polygon meshes is important for real time applications.

The authors want to thank the Stanford Computer Graphics Laboratory for providing us through their web site http://www-graphics.stanford.edu/data/3Dscanrep, with the range data we used for our experiments.

\section{[References]}

1) S.Rusinkiewics, O.H.Holt, M.Levoy,"Real time 3D Model Acquisition", ACM Siggraph2002, san Antonio (July2002)

2) J.C.Carr, R.K.Beatson, J.B.Cherrie, W.R.Fright, B.C.McCallum. "Reconstruction and Representation of 3D Objects with Radial Basis Functions", ACM Siggraph 2001, pp 67-76 (August 2001)

3) Bryan S.Morse, Terry S.Yoo, Penny Rheingans, David T.Chen K.R.Subramanian. "Interpolating Implicit Surfaces From Scattered Surface Data Using Compactly Supported Radial Basis Functions", Shape Modelling International, Itally (May 2001)

4) R.K.Beatson, J.B.Cherrie, D.L.Ragozin,. "Fast Evaluation of Radial Basis Functions: Methods for Four Dimensional Polyharmonic Splines", SIAM J.Sci., 32, 6, pp1272-1310 (2001)

5) R.K.Beatson,W.A.Light, S.Billings, "Fast Solution of the Radial Basis Function Interpolation Equations: Domain decomposition Methods", SIAM J.Sci. 22, 5, pp1717-1740 (2000)

6) Braga, A. P., Carvalho, A. C. P. L. F., Ludermir, T. B., Almeida, M.B. and Lacerda, "Radial Basis Functions: Theory and Applications," Nonlinear Modeling and Forecasting of High Frequency Financial and Economic Time Series, Kluwer Publications. In Press.

7) P.Alliez, M.Meyer, M.Desbrun. "Interactive Geometry Remeshing", ACM SIGGRAPH'2002 Conference Proceedings, 21, pp347354 (July 2002)

8) S.Rusinkiewicz, O.Hall-Holt, M.Levoy. "Real-Time 3D Model Acquisition", ACM Siggraph 2002, 21, pp438 - 446 (July 2002)

9) D.K.Pai, K.Van den Doel, D.L.James, J.E.Lloyd, J.L.Richmond, S.H.Yau. "Scanning Physical Interaction Behavior of $3 \mathrm{D} \mathrm{Ob}$ jects", ACM Siggraph 2001, 28, pp87 - 96 (August 2001)

10) T.Poston, T.T.Wong, P.A.Heng, "Multiresolution Isosurface Extraction with Adaptive Skeleton Climbing", Eurographics'98, 17, 3 (1998)

11) G.M.Treece, R.W.Prager, A.H.Gee, "Regularised Marching Tetrahedra: improve isosurface extraction", CUED/F-INFENG/TR 333 Computers and Graphics, pp583-598 (Sept. 1999)

12) Morgan Kaufmann, "Introduction to Implicit Surfaces", J.Bloomenthal, editor, San Francisco, California (1997)

13) Shigeru Muraki, "Volumetric shape description of range data using Blobby Models", Proceeding of Siggraph 91, 25(4), pp.227235 (1991)

14) Desbrun, M., M. Meyer, P. Schroder, and A.H. Barr. "Implicit Fairing of Irregular Meshes Using Diffusion and Curvature Flow". SIGGRAPH Proceedings, pp317-324 (Agust 1999) 


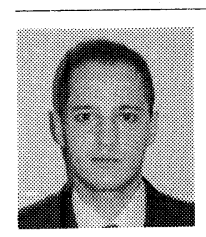

Hamid Laga was born in Algiers, Algeria on October 15, 1974. He received the Bachelor degree of Computer Science from the Institut National d'Informatique INI, Algiers, Algeria in 1997. He was a research student at the Computer Science department, the Graduate School of Information Science and Engineering, Tokyo Institute of Technology, Japan, from April 2000 until March 2001, where he received the Master degree of Computer Sciecne in 2003. He is now a candidate for a Doc tor degree of Engineering at the same department. His fields of interest include computer vision, 3D reconstruction and computational geometry.

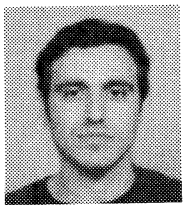

Romanos Piperakis was born in Athens, Greece on August 29, 1975. He received his diploma from the university of Patras, Greece in 1997 and his Doctor of Engineering in the department of computer science of Tokyo Institute of Technology, Japan, in 2002. Currently he is working as a researcher for the precision and intelligence laboratory at Tokyo Institute of Technology. His fields of interest include realistic human animation, virtual reality, autonomous intelligent agents and neural networks.

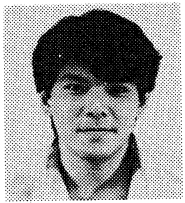

Hiroki Takahashi was born in Hokkaido Japan on February 25, 1967. He received the B.E degree and the ME degree from the Tokyo Institute of Technology, Japan in 1990 and 1992 respectively. Since 1994 he has been with the department of Computer Science, the Graduate School of Information Science and Engineering, Tokyo Institute of Technology, Tokyo, Japan, where he is now a Research Associate. His fields of interests are pattern recognition, neural networks and genetic algorithms.

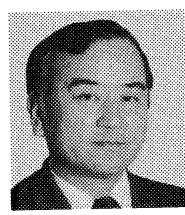

Masayuki Nakajima was born in Tokyo, Japan, on August 3, 1946. He received the B.E.E degree and Dr.Eng. degree from the Tokyo Institute of Technology, Japan, in 1969 and 1975, respectively. Since 1975 , he had been with the Department of Imaging Science and Engineering, Tokyo Institute of Technology, Japan. He is now a professor at the Department of Computer Science, the Graduate School of Information Science and Engineering, Tokyo Institute of Technology, Japan. His fields of interest are computer graphics, pattern recognition, image processing and virtual reality. 